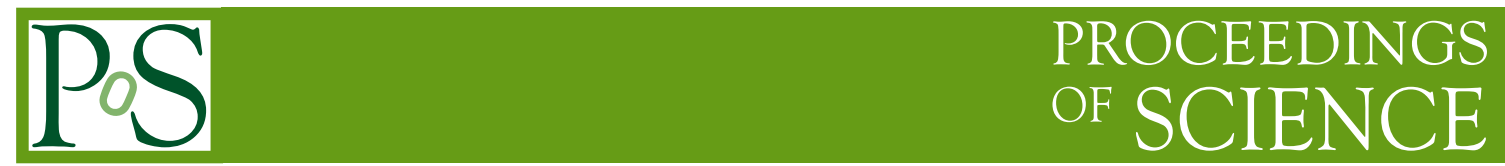

\title{
UV-filtered overlap fermions
}

\section{Stephan Dürr}

Bern University

E-mail: durr (AT) itp.unibe.ch

\section{Christian Hoelbling*}

Wuppertal University

E-mail: hoelbling (AT) physik.uni-wuppertal.de

\section{Urs Wenger}

NIC/DESY Zeuthen

E-mail: urs.wenger (AT) desy.de

We discuss the kernel spectrum, locality properties and the axial-vector renormalization constant of UV-filtered overlap fermions. We find that UV-filtered overlap fermions have a better conditioned kernel, better locality and an axial-vector renormalization constant closer to 1 than their unfiltered counterparts, even if the shift parameter $\rho$ is simply set to 1 .

XXIIIrd International Symposium on Lattice Field Theory

25-30 July 2005

Trinity College, Dublin, Ireland

${ }^{*}$ Speaker. 


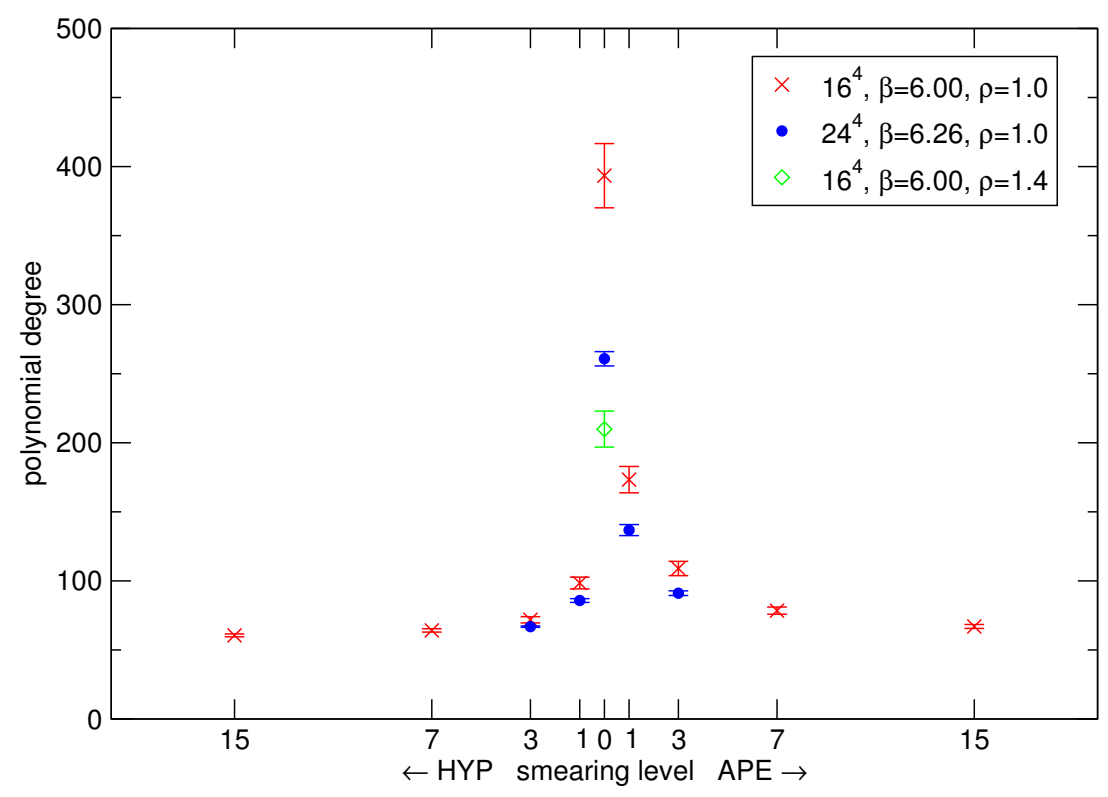

Figure 1: Degree of the Chebychev polynomial required to achieve a minimax accuracy of $10^{-8}$ on a $16^{4}$ lattice at $\beta=6.0$. With $\rho=1$ a single HYP step brings for the massless overlap operator a speedup factor $\sim 4$, but a fair comparison is to the situation with $\rho=1.4$ without filtering, and then the factor is $\sim 2$.

\section{Introduction}

Overlap fermions [1] have many theoretically desirable features, but they are computationally rather demanding. There are suggestions in the literature on how to reduce the computational cost by using a more elaborate kernel together with fattened gauge links [2, 3, 4]. More recently, it has been observed that UV-filtering alone has a similar effect $[5,6,7,8,9,10]$ and maintains the $O\left(a^{2}\right)$ Symanzik class. In this note we investigate the effectiveness of UV-filtering in the quenched approximation. We use the Wilson gauge action and employ APE [11] or HYP [12] smearing. For details of the implementation and simulation parameters we refer the reader to $[9,10]$.

\section{Kernel spectrum}

The overlap operator is constructed from the hermitean Wilson operator $H_{\mathrm{W}, m}=\gamma_{5} D_{\mathrm{W}, m}$ via

$$
D_{\mathrm{ov}}=\rho\left(1+\operatorname{sign}\left(H_{\mathrm{W},-\rho}\right)\right)
$$

where $\rho$ is the shift parameter. In a practical implementation one has to find a method to approximate the sign function in (2.1) over the entire eigenvalue spectrum of $H_{\mathrm{W},-\rho}$. Therefore, it is essential for an efficient implementation that the kernel operator $H_{\mathrm{W},-\rho}$ is well conditioned.

In the free theory the condition number $C(\rho)$ of $\left|H_{\mathrm{W},-\rho}\right|$ is given by

$$
C(\rho)= \begin{cases}\frac{8-\rho}{\rho} & \text { for } 0<\rho \leq 1 \\ \frac{8-\rho}{2-\rho} & \text { for } 1 \leq \rho<2\end{cases}
$$

which has a minimum value 7 at $\rho=1$. In the interacting theory the upper edge of the spectrum at $8-\rho$ is only slightly affected for typical $\beta$. On the other hand the absence of a lower bound on the 

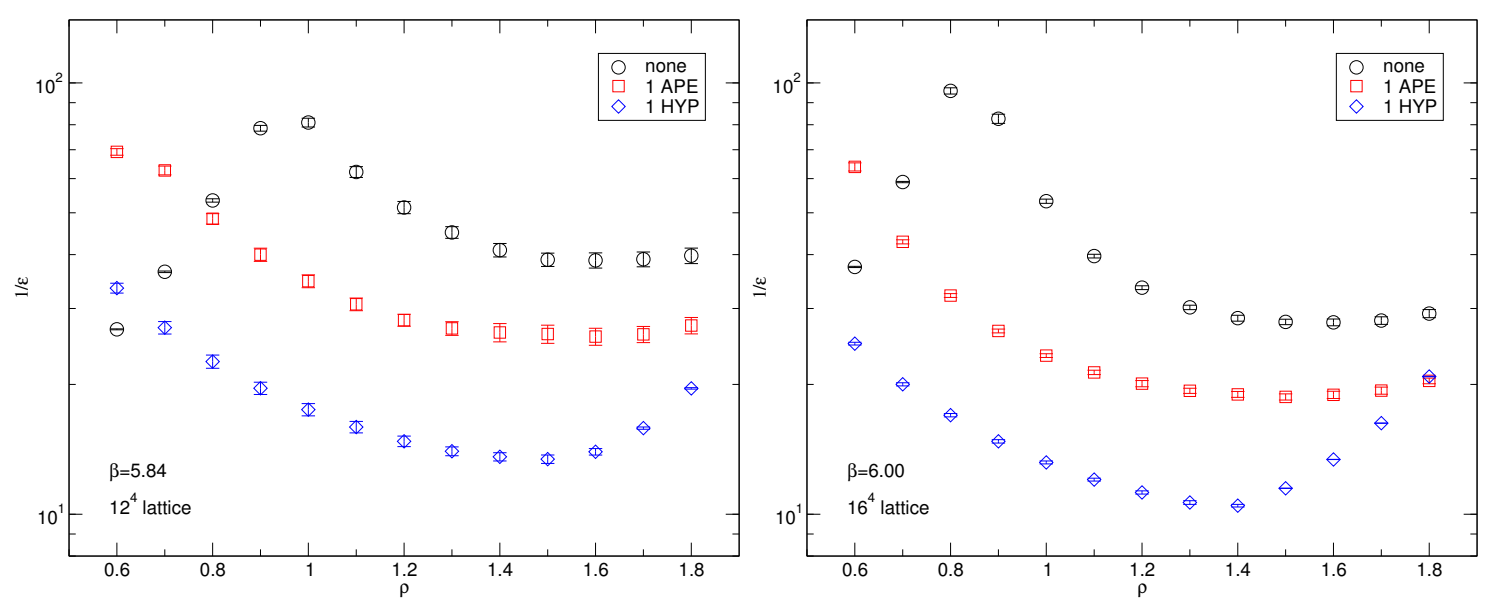

Figure 2: Condition number $1 / \varepsilon=C(\rho)$ of $\left|H_{\mathrm{W},-\rho}\right|$ versus $\rho$ for $\beta=5.84$ (left) and $\beta=6.0$ (right).

spectrum can result in a dramatic increase of the condition number $C(\rho)$. Since there are standard techniques to treat the lowest few of these eigenmodes exactly, the relevant issue is the onset of the bulk of these modes, and it turns out that the latter gets significantly lifted by UV-filtering [9].

In order to translate the condition number into computational cost, we choose to present the degree of the Chebychev polynomial [13] necessary to reach a given precision in the minimax norm after treating the 14 lowest eigenmodes exactly. As one can see from Fig. 1, the number of forward applications of $H_{\mathrm{W}}$ needed to obtain a given precision is significantly reduced by UV-filtering.

More details are shown in Fig. 2. A rather conservative filtering recipe like applying 1 step of APE link fattening seems to render the kernel far more benevolent; the condition number remains comfortably small for a large range of shift parameters, including the canonical choice $\rho=1$.

\section{Locality}

The "spread" inherent in the gauge link fattening raises the question whether a UV-filtered overlap operator is less local than the original thin link version. It has already been observed in [6] that the UV-filtering actually proves beneficial to the locality properties of the overlap operator.

Fig. 3 shows the localization of the overlap operator at $\beta=6.0$. Following the convention of [14] we plot the maximum over the 2-norm of $D_{\mathrm{ov}} \eta$ at $x$ with $\eta$ a normalized $\delta$-peak source vector at the point 0 in the lattice versus the "taxi driver" distance $d_{1}=\|x\|_{1}$ to the location of the $\delta$-peak

$$
f\left(d_{1}\right)=\sup \left(\left\|\left(D_{\mathrm{ov}} \eta\right)(x)\right\|_{2} \mid\|x\|_{1}=d_{1}\right) .
$$

Looking first at the unfiltered operators (black/dark diamonds and crosses) one finds the well known result that (at this $\beta$ ) adjusting $\rho$ to a value around 1.4 lets $f\left(d_{1}\right)$ fall off steeper than keeping the untuned value $\rho=1$. The interesting observation is that a single HYP step together with $\rho=1$ (red/light squares) results in an even steeper descent than the unfiltered version with $\rho=1.4$ (which was chosen to nearly optimize the locality of the unfiltered operator). The last curve shown (red/light pluses) indicates that one should not attempt to combine the filtering with a $\rho$ value that would be optimal for the unfiltered operator. 


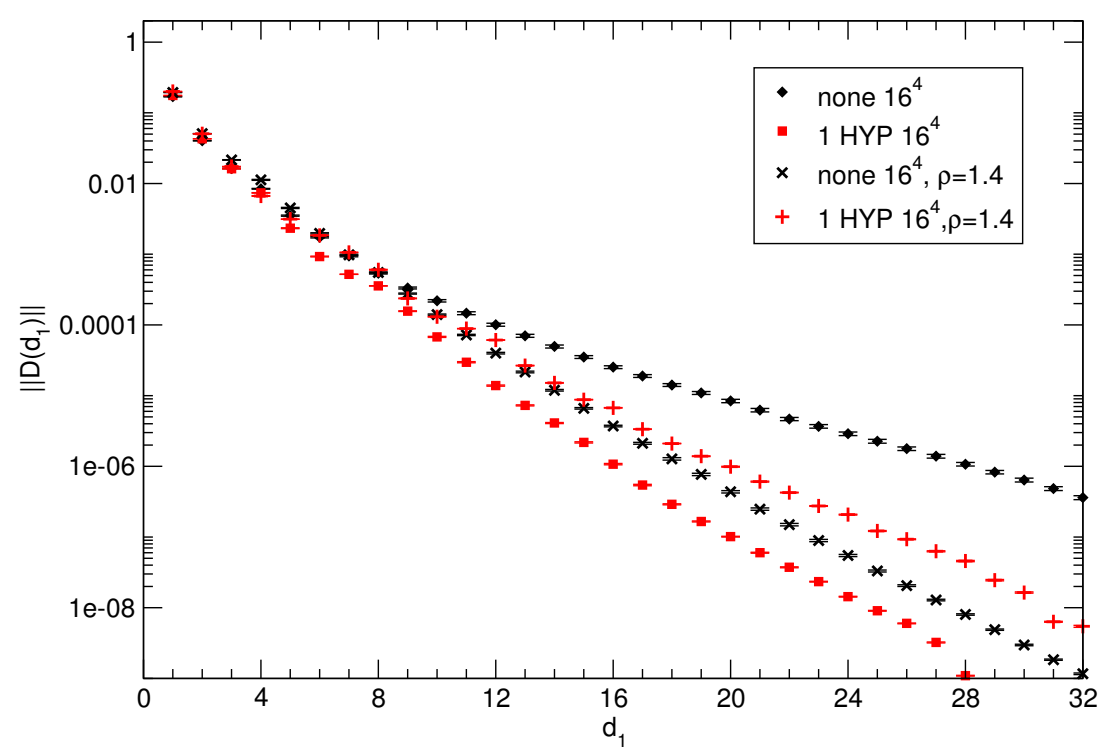

Figure 3: Localization of the overlap operator at $\beta=6.0$ without filtering and after 1 HYP step, for $\rho=1$ and $\rho=1.4$. A single HYP step proves more efficient than optimizing $\rho$. Filtering and $\rho>1$ should not be combined; for the filtered operator the untuned choice $\rho=1$ is reasonable (but still not optimal).

\section{Renormalization}

One interesting aspect of UV-filtered fermions is their improved renormalization behavior. It has been realized some time ago $[15,16]$ that in general one-loop corrections to renormalization constants are substantially smaller in the case of UV-filtered fermions.

To investigate this behavior in a nonperturbative context we choose to determine the axialvector renormalization constant $Z_{A}$ from the AWI, following the method of $[17,18]$. Our results are collected in Tab. 1 [9]. It is interesting to note that for the untuned $\rho=1$ thin link operator $Z_{A}$ is large even at $\beta=6$. With $\rho=1.4$ and the same coupling $Z_{A}$ is much closer to 1 , indicating that $\rho>$ 1 is not only essential for the locality of the unfiltered $D_{\text {ov }}$ but also beneficial to its renormalization constants. Note, however, that even the 1-fold APE smeared operator with the untuned $\rho=1$ has an axial-vector renormalization constant which is closer to 1 . When going to the coarser $\beta=5.84$ lattice $Z_{A}$ of the thin link operator with $\rho=1$ increases drastically. At this point it becomes essential

\begin{tabular}{|l|cccc|}
\hline & 5.66 & 5.76 & 5.84 & 6.00 \\
\hline$Z_{A}^{\text {none }}, \rho=1$ & ill-def. & ill-def. & $7.06(73)$ & $3.145(94)$ \\
$Z_{A}^{\text {none }}, \rho=1.4[19]$ & - & - & $\sim 1.71$ & $1.553(02)$ \\
$Z_{A}^{\text {none }}, \rho=1.6[20]$ & - & - & $\sim 1.44$ & - \\
$Z_{A}^{1 \mathrm{APE}}, \rho=1$ & $2.57(7)$ & $1.90(2)$ & $1.66(02)$ & $1.452(04)$ \\
$Z_{A}^{3 \mathrm{APE}}, \rho=1$ & $1.55(3)$ & $1.33(1)$ & $1.23(01)$ & $1.160(06)$ \\
$Z_{A}^{1 \mathrm{HYP}}, \rho=1$ & $1.44(2)$ & $1.28(1)$ & $1.22(01)$ & $1.153(03)$ \\
$Z_{A}^{3 \mathrm{HYP}}, \rho=1$ & $1.21(1)$ & $1.13(1)$ & $1.10(01)$ & $1.072(02)$ \\
\hline
\end{tabular}

Table 1: $Z_{A}$ with several filterings. Note that $[19,20]$ give $Z_{A}$ values at slightly different $\beta$ only. The precise numbers are $Z_{A}^{\text {none }}(\beta=5.8458, \rho=1.4)=1.710(5)$ [19] and $Z_{A}^{\text {none }}(\beta=5.85, \rho=1.6)=1.443(5)$ [20]. 


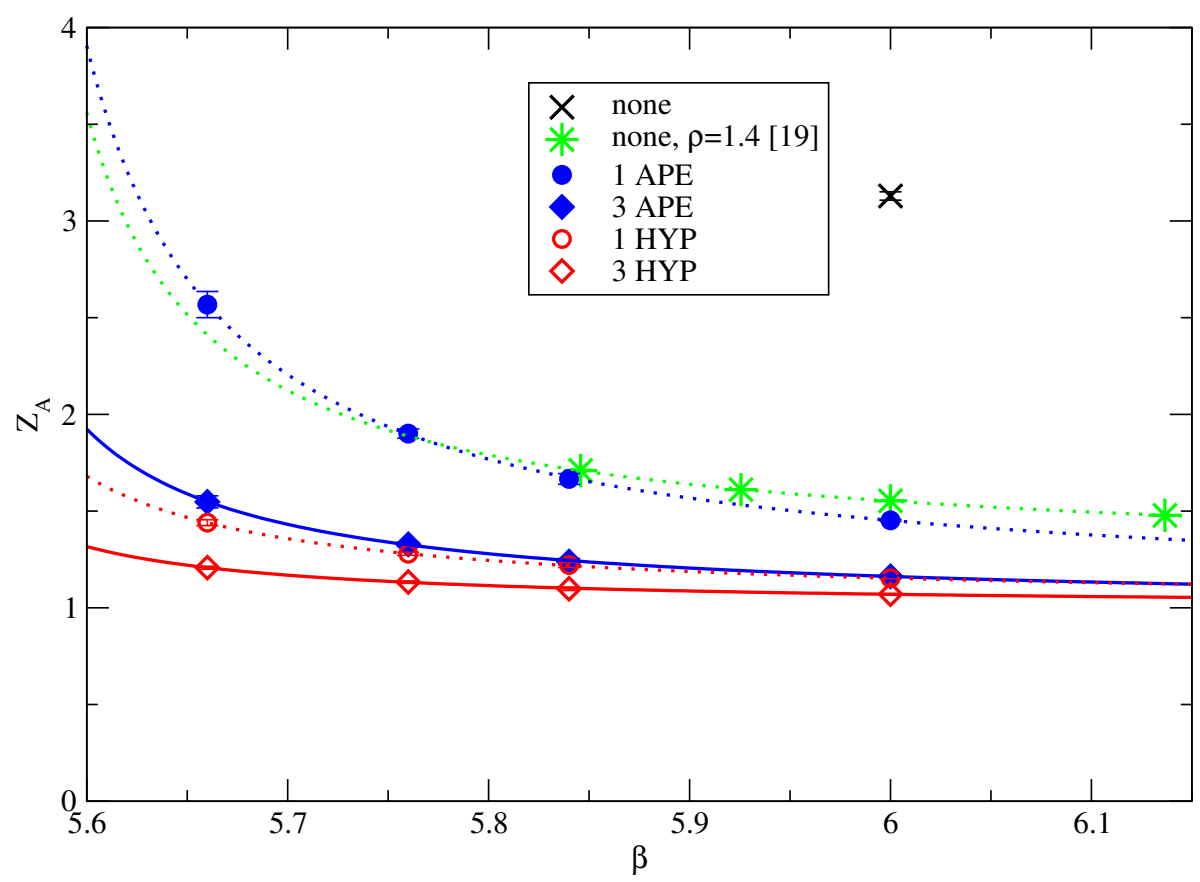

Figure 4: Axial-vector renormalization constant $Z_{A}$ versus $\beta$. The thin link data at $\rho=1.4$ are from [19].

\begin{tabular}{|l|ccccc|}
\hline filtering level & none, $\rho=1.4[19]$ & $1 \mathrm{APE}$ & $3 \mathrm{APE}$ & $1 \mathrm{HYP}$ & $3 \mathrm{HYP}$ \\
\hline$c_{1}+c_{3}$ & $1.66(16)$ & $0.36(32)$ & $0.03(20)$ & $0.15(15)$ & $0.03(13)$ \\
$c_{3}$ & $5.54(4)$ & $5.53(2)$ & $5.51(4)$ & $5.49(4)$ & $5.49(5)$ \\
\hline
\end{tabular}

Table 2: The 1-loop coefficient $c_{1}+c_{3}$ and pole location $c_{3}$ of a fit (4.1) for different levels of UV-filtering. Note, that the analytic value for $c_{1}+c_{3}$ for thin link overlap at $\rho=1.4$ is $c_{1}+c_{3}=0.722408$ [21, 22].

for the thin link operator to properly tune $\rho$. Going to even coarser lattices we were unable to obtain a signal for $Z_{A}$ with the unfiltered operator and $\rho=1$ (for details see [9]).

In Fig. 4 the $Z_{A}$ with different filterings are plotted vs. $\beta$. The lines represent fits of the form

$$
Z_{A}(x)=\frac{1+c_{1} x+c_{2} x^{2}}{1-c_{3} x}
$$

with $x=1 / \beta$ and all fits have a reasonable $\chi^{2}$. To compare to the thin link $\rho=1.4$ case we use data from [19]. In principle, these curves contain two pieces of information. The asymptotic slope $c_{1}+c_{3}$ for $x \rightarrow 0$ predicts the perturbative 1-loop coefficient for $Z_{A}$ and the pole in (4.1) [i.e. the value of $c_{3}$ ] predicts the coupling where the ansatz (4.1) breaks down. With the current quality of our data all asymptotic slopes - except for the thin link operator - are compatible with zero and we are unable to make a quantitative statement about the perturbative 1-loop coefficients (see Tab. 2). Still, on a qualitative level the filtered one-loop coefficients are smaller than both the analytic result and the fit to nonperturbative data for thin link overlap with $\rho=1.4$. This suggest a much better behaved perturbative series for the filtered overlap operator [9]. For the location of the pole the results in Tab. 2 indicate that this quantity is hardly changed by the filtering. This suggests that the (practical) range of validity of the perturbative expansion is barely affected by the filtering [9]. 


\section{Conclusions}

We have presented some evidence that UV-filtered overlap fermions might bring substantial technical advantages over their thin link counterparts. In particular, a forward application of the massless overlap operator requires fewer applications of the hermitean Wilson operator, the locality of the overlap operator is generally improved with UV-filtering and the axial-vector renormalization constant is much closer to 1 . In addition, there is no need to tune the kernel shift parameter $\rho$. The canonical choice $\rho=1$ is satisfactory even on rather coarse lattices.

\section{References}

[1] H. Neuberger, Phys. Lett. B 417141 (1998) [hep-lat/9707022].

[2] W. Bietenholz and I. Hip, Nucl. Phys. B 570, 423 (2000) [hep-lat/9902019].

[3] W. Bietenholz, Nucl. Phys. B 644, 223 (2002) [hep-lat/0204016].

[4] T. DeGrand [MILC Collab.], Phys. Rev. D 63, 034503 (2001) [hep-lat/0007046].

[5] W. Kamleh, D.H. Adams, D.B. Leinweber and A.G. Williams, Phys. Rev. D 66, 014501 (2002) [hep-lat/0112041].

[6] T.G. Kovacs, Phys. Rev. D 67, 094501 (2003) [hep-lat/0209125].

[7] T. DeGrand, A. Hasenfratz and T.G. Kovacs, Phys. Rev. D 67, 054501 (2003) [hep-lat/0211006].

[8] S. Dürr, C. Hoelbling and U. Wenger, Phys. Rev. D 70, 094502 (2004) [hep-lat/0406027].

[9] S. Dürr, C. Hoelbling and U. Wenger, JHEP 0509, 030 (2005) [hep-lat/0506027].

[10] S. Dürr and C. Hoelbling, hep-ph/0508085.

[11] M. Albanese et al. [APE Collab.], Phys. Lett. B 192, 163 (1987).

[12] A. Hasenfratz and F. Knechtli, Phys. Rev. D 64, 034504 (2001) [hep-lat/0103029].

[13] P. Hernandez, K. Jansen and L. Lellouch, hep-lat/0001008.

[14] P. Hernandez, K. Jansen and M. Lüscher, Nucl. Phys. B 552, 363 (1999) [hep-lat/9808010].

[15] C.W. Bernard and T. DeGrand, Nucl. Phys. Proc. Suppl. 83, 845 (2000) [hep-lat/9909083].

[16] T. DeGrand, Phys. Rev. D 67, 014507 (2003) [hep-lat/0210028].

[17] M. Lüscher, S. Sint, R. Sommer and P. Weisz, Nucl. Phys. B 478, 365 (1996) [hep-lat/9605038].

[18] L. Giusti, C. Hoelbling and C. Rebbi, Phys. Rev. D 64, 114508 (2001) [Erratum-ibid. D 65, 079903 (2002)] [hep-lat/0108007].

[19] J. Wennekers and H. Wittig, JHEP 0509, 059 (2005) [hep-lat/0507026].

[20] R. Babich et al., hep-lat/0509027.

[21] C. Alexandrou, E. Follana, H. Panagopoulos and E. Vicari, Nucl. Phys. B 580, 394 (2000) [hep-lat/0002010].

[22] S. Capitani and L. Giusti, Phys. Rev. D 62, 114506 (2000) [hep-lat/0007011]. 